\title{
Magnetic Microfluidic Linear Halbach Array Configuration for Cell Separation
}

\author{
Omer Saeed $^{12}$, lucienne Duru ${ }^{1}$ and Deng Yulin ${ }^{1}$ \\ ${ }^{1}$ School of Life Science, Département of Biomédical Engineering, Beijing Institute of Technology, Beijing 100081, China. \\ ${ }^{2}$ Faculty of science and Technology, Department of Biomedical Physics, ALNeelain University, Khartoum 1121, Sudan.
}

\begin{abstract}
The different type of blood entities like red blood cells (RBCs), white blood cell (WBCs) and platelets counts is a critical point in routine medical tests. Circulating tumor cells (CTCs) counting and analysing in the blood is indicative of the stage and origin of cancer in particular time. Here we are describing a proposed microfluidic device, used a magnetic means for CTCs separation from a blood or buffer sample. The device characterizations were simulated using COMSOL MULTIPHYSICS software as well as practically validated. Using a known spiked number of CTCs in the sample, the purity and recovery rate were calculated to be $93 \%$ and respectively.
\end{abstract}

\section{Introduction}

Particle separations are actual particulate that are not dissolved in the sample but rather than particles are suspended in the sample, these samples would be referred as suspensions. There are a lot of different application of particle sorting and counting in blood analysis and environmental field. In fact one of the big areas in this field now is the ability to capture and sort circulating tumor cells (CTCs) in the blood. It turns out that the CTCs have slightly different properties than normal cells, they are more rigid and less squishy than the normal cells are[1].

Circulating tumor cells (CTCs) are thought to be cells that have been shed from a primary tumor and have transferred into the circulation system, those cells then have the potential to form a metastatic in the patient so the cells are very dangerous to the patient more so than once in the primary tumour because in most patients the mortality comes from metastatic cancer not from the primary tumour. These cells are really poorly defined as we really feel that we don't have the best definition of what a circulating tumour cell is, in order to capture a heterogeneous set of CTCs. the current definition most people agree is that given by cell search which is the only F.D.A. cleared method for enumerating. And they define a circulating tumor cell as a large nucleated cell, with a positive staining for EpCAM (Epithelial Cell Adhesive Molecules) and cytokeratin 8,18 or 19 which is the epithelial cells intercellular marker and negative for leukocyte marker staining CD45. These cells are very difficult to study because they're in such low abundance, in one $\mathrm{ml}$ of whole blood there may be ten million white blood cells (WBCs) and five billion red blood cells (RBCs) while in a metastatic in a breast cancer patient may have on the order of one hundred circling tumour cells, so they're very difficult to separate them because of their extremely low abundance. We're interested in studying these because they hold such promise of prognostic value to the patient.

There are many researcher have studied the cells deformability and being able to sort different cells types based on those parameters[2][3]. Also there is some work that has been published earlier is well known now, that the cancer cells have slightly different size than normal cells[4] . So it's possible to separate cancer cells from noncancerous ones based on size with very high resolution.

Most recently, that have actually been shown that cancer cells have express different types of membrane protein than normal cells do, called surface markers, created pillar like structures functionalized antibodies groups on the wall will capture pushed cells and protein as they passed by those pillars. There are a lot of interests in counting and sorting the different types of particles in blood, either they are RBCs, WBCs cancerous and noncancerous cells[5][6]. Cells counting are very important in medical diagnostics. Another example of cell counting in Human Aids Virus detection (HIV) diagnostic, where CD4 cells number in blood is an indicative of positive or negative diagnose[7]. All these techniques of particle and cell counting required methods to capture and sort particles in a liquid for sorting based on different properties. Besides blood analysis, also particle sorting has many applications in environmental and biology fields. Such in water monitoring, as a water sample may contains different types of organisms ranging from single cell organisms and bacteria, separating and counting these different life species will acts as environmental monitoring detector[8]. Cell sorting and focusing 
techniques are ultimately applied to flow cytometer concepts. A flow cytometer is a method for counting and differentiating cell types. The idea is that cells will flows in a single file through a window where they are detected by a laser beam. The cells suspension sample is combined with two other outsides liquid flows form a parallel streams through the length of the channel, called hydrodynamic techniques or sheath flows[9].

Basically we're interested in capturing a heterogeneous subset of circulating tumor cells, using a simple magnetic means technology that allows us to isolate a single circulating tumor cells that are appropriate for downstream analysis. We fabricated and test a microfluidic chip with permanent magnets linear array located at a distance from the channel wall to generate the magnetic field required to deflect and alter the magnetic particle and so magnetic particle labelled cell to the specific determined outlet. The collected cells sample has been characterized using flow cytometer and fluorescent microscope to determine the fabricated microdevice ability in cells separation.

\section{Merterial and methods}

The microfluidic device is simplify fabricated using PDMS with glass slide followed the standard photolithography process. The basic principle of the operation is relatively straight forward, part of particles could be magnetized and magnetophoresis is basically means a flow in the channel. In the figure .1 (A) below as the flow going from right to left, a magnetic field that's acting on the particles. The magnetic field can cause the particles to migrate at different rates depending on their size and magnetic properties. Here the sample mixture is going through the middle streamline. The sample is coming down the middle part of the channel and the others are just buffers. As continuous free flow electrophoresis, without magnetic field all the particles will just travel straight through the bottom, but with magnetic field different magnetized particles will travel at different speeds upwards depending on their properties. So the different types of particles would end up at different outlets.

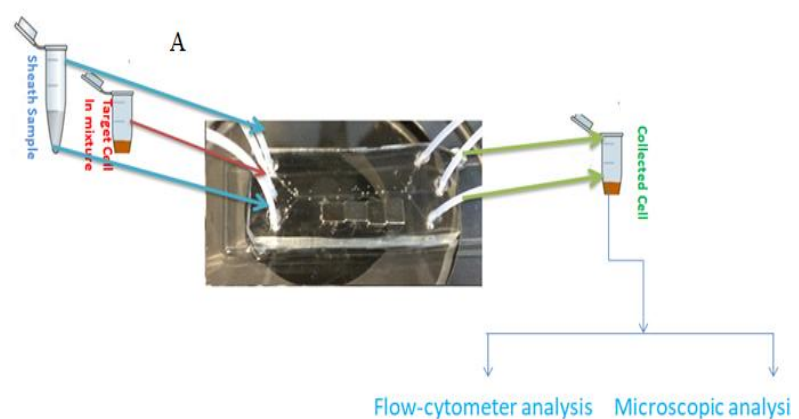

\section{B}

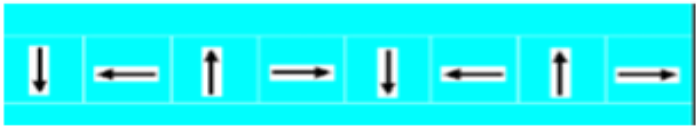

Figure 1. (A) Working and characterization steps of the proposed device. (B) The magnets orientation used.
Firstly, the magnetic field simulation of linear Halbach arrays is carried out by using COMSOL finite element software. The distribution of magnetic flux and the distribution of magnetic field are obtained. From these results, it can be seen that the Halbach array has the characteristics of one side magnetic field and the other side is very small. This feature lays a theoretical foundation for the development of various magnetic devices made up of Halbach arrays. The traditional linear Halbach arrays used her is shown in figure .1 (B). We used eight $3 \mathrm{~mm} 3$ cubic permanent magnets, with specific orientation as in figure.1(B).

To represent what we would see in a patient sample we've done spiking experiments, where we spiked the chosen MCF7 Cells line into whole blood. Then ficoll gradient was performed to obtain a Buffy coat. Lastly we positively labelled the MCF7 Cell in the sample with anti-Epcam antibodies that have a magnet bead attached to it. The sample contained the labelled MCF7 is followed into our microfluidic device through the middle inlet using a syringe pump (Cole-Parmer, Vernon Hills, IL, USA) with a specific flow rate. The schematic graph of the sample preparation is shown in figure .2 below

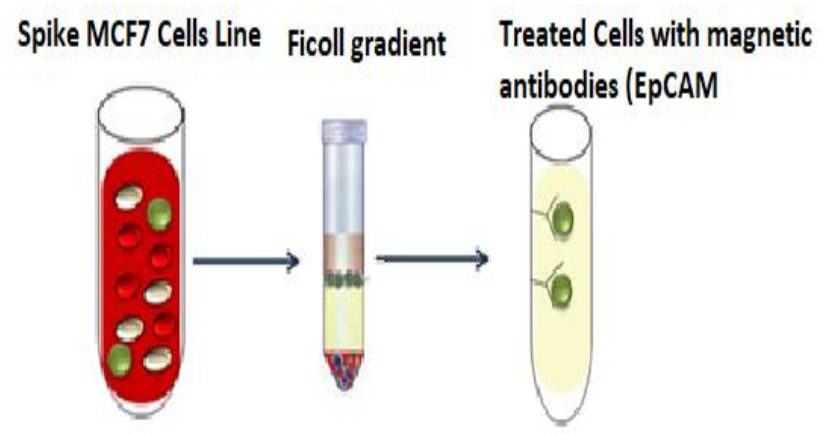

Figure 2. the sample preparations steps.

In order to solve the magnetic field distribution of the Halbach array, we have to solve the Maxwell equation by the basic theory of the electromagnetic field. The partial differential equation and boundary conditions of the magnetic field can be obtained from the Maxwell equation:

$$
\begin{aligned}
& \nabla \cdot\left(\mu_{0} \mu_{r} \mathrm{H}\right)=0 \\
& \qquad 56^{H}=-\nabla V_{m}
\end{aligned}
$$

Where $\mu 0$ and $\mu \mathrm{r}$ are the vacuum and material relative permeability respectively. $\mathrm{H}$ and $\mathrm{V}$ is the magnetic and potential field respectively.

\section{Results and discussion}

The designed three inlets and outlets microchannel,s width (w) and length (L), used here in this analysis were $200 \mu \mathrm{m}$ and $30 \mathrm{~mm}$ in $\mathrm{y}$ and $\mathrm{x}$ direction respectively. 
To determine the magnetic field distribution in the configuration above, a $2 \mathrm{D}$ model was constricted and simulated using Finite Element Method (FEM) package COMSOL. The simulation model includes the polydimethylsiloxane (PDMS) channel, the permanent magnets array and the flow media, as shown in Figure. 1. In order to limit the simulation domain, outer circle was used. The array contains eight of $3 \mathrm{~mm} 3$ cubic shape and 49600 A.m saturation magnetization $\mathrm{NdFeB}$ magnet was used. Theses magnets was placed at $2 \mathrm{~mm}$ away from the channel wall.

Magnetic field strength $H_{v}$ in both channel walls (at $\boldsymbol{y}=\mathbf{0}$ and $\boldsymbol{H}$ ) was computed, so as the magnetic field gradient in $\mathrm{y}$ direction $\frac{\partial \boldsymbol{H}_{y}\left(\boldsymbol{x}_{i} \boldsymbol{y}\right)}{\mathrm{a}_{x}}$ throughout the microchannel width has been estimated to be 592.813 $\mathrm{A} / \mathrm{m}$.

The resulted magnetic field distribution is shown in figure 3 below
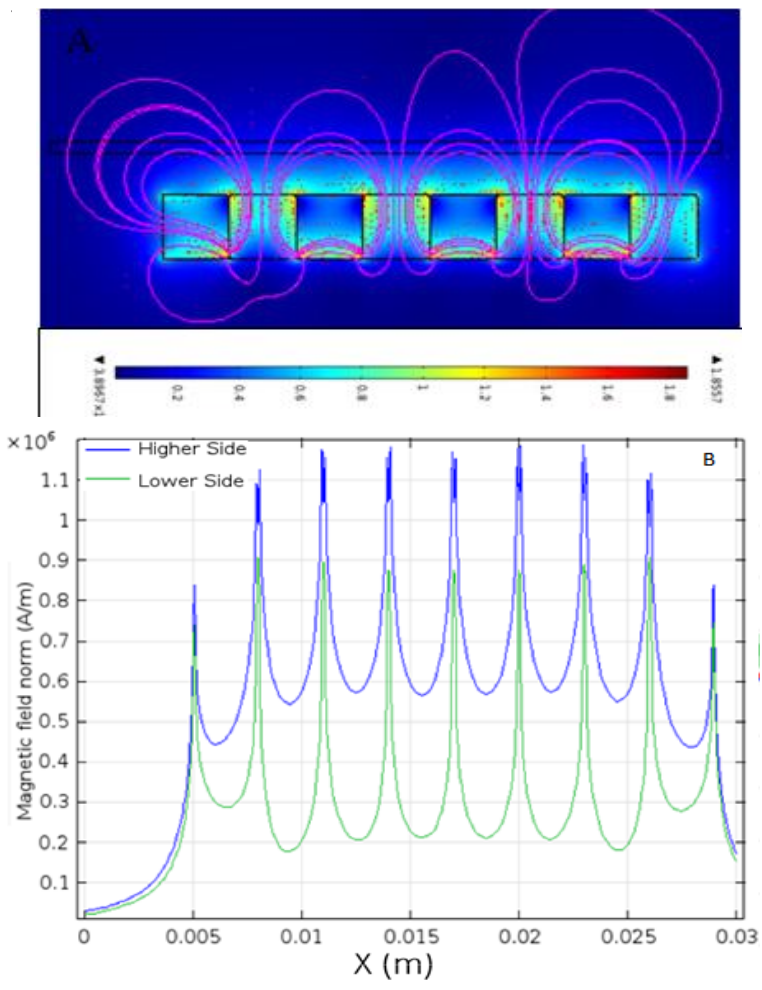

Figure 3. Magnetic field generated from the linear magnets array and the michrochannel location relative to the magnets. (A) Surface plot. (B) Magnetic field along the $\mathrm{X}$ axis at the surface of the magnets on higher and lower sides of the array.

Additionally the magnetic field gradient components $\mathrm{X}$ and $\mathrm{Y}$ have been calculated at different distance from the array surfaces, particularly, at 1,2 and $2.250 \mathrm{~mm}$ and shown in figure 4 below.

The magnetic forces in both $\mathrm{X}$ and $\mathrm{Y}$ directions, that acting on the beads is given by[10]

$$
F_{\text {mag. } x}=\frac{4 \pi r_{p}{ }^{3} \Delta \chi}{3 \mu_{0}} B_{x} \frac{\partial B_{x}(x, y)}{\partial x}
$$

$$
F_{\text {mag, } y}=\frac{4 \pi r_{p}^{3} \Delta \chi}{3 \mu_{0}} B_{y} \frac{\partial B_{y}(x, y)}{\partial y}
$$

Where $\mathrm{B}=$ the magnetic field, $\mu 0=$ the permeability of the space, $\Delta \mathrm{x}=$ magnetic susceptibility of the bead and that of the fluid difference and $\mathrm{rP}=$ radius of the bead magnetic core.

The magnetic beads acting force components $\mathrm{X}$ and $\mathrm{Y}$ were solved numerically and given in figure. 5. From there it's obviously that, the $\mathrm{Y}$ component of the force is much greater than the $\mathrm{X}$ one, with higher negativity, indicates the attractive force strength on the magnetic beads and affects their path with a displacement in y direction along the microchannel width, and end up through the desired exit.
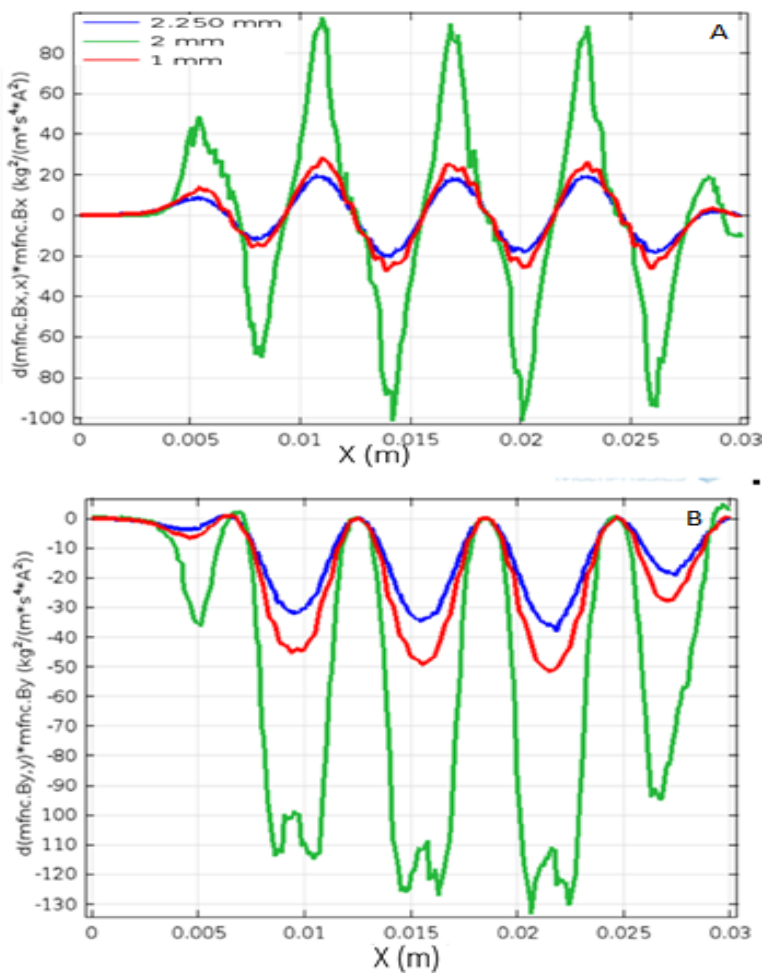

Figure 4. Magnetic field gradients. (A)X component. (B) Y component.

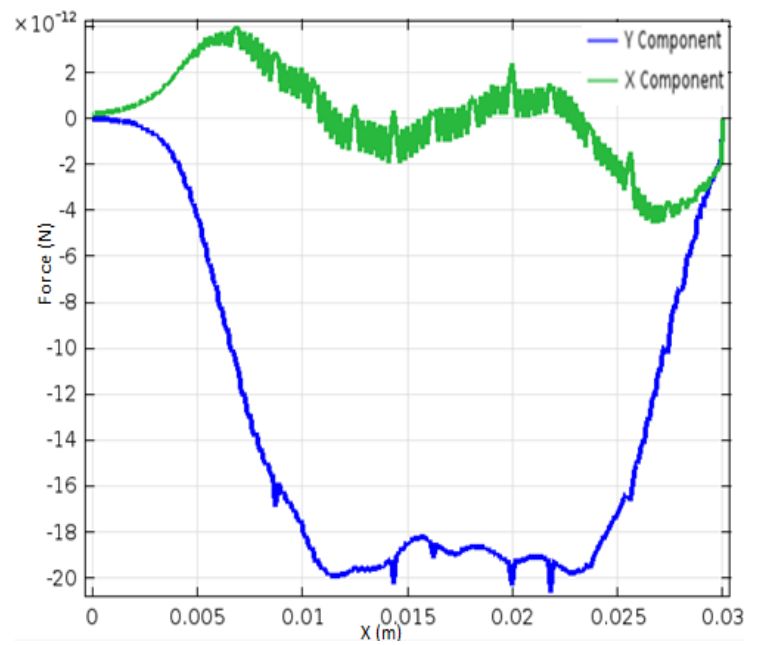

Figure 5. the $\mathrm{X}$ and $\mathrm{Y}$ magnetic acting force. 
Magnetic particle trajectories for time saving, was simulated only in the main channel domain. This domain has the same dimension parameters of the channel (200 um and $30 \mathrm{~mm}$ ) high and length respectively.

The magnetic particle trajectories under magnetic field effects were simulated numerically as shown in figure .6. It's clearly shown that, the magnetic beads followed a $15 \mathrm{~mm}$ distance inside the microchannel without suffering any displacement, but after this point (tip of the magnets array) they start to deflect from their original path as here the magnetic force is greater than the fluidic drag force.
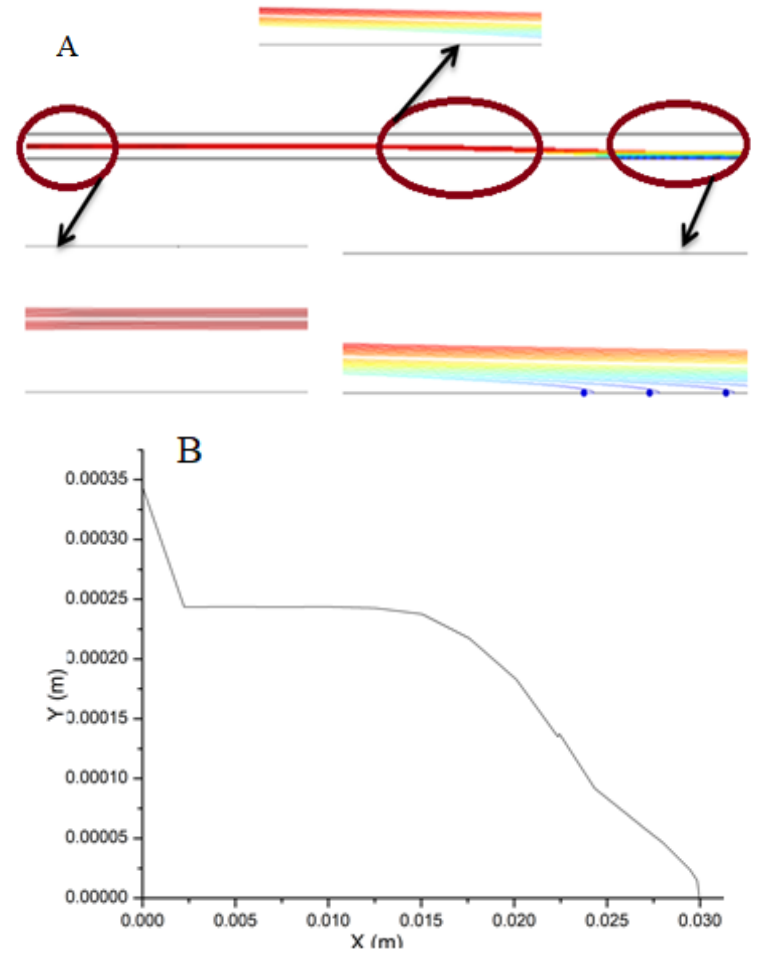

Figure 6. (A) snap shot images of the magnetic beads trajectories. (B) Beads trajectories across the channel width.

Experimentally, we tested the separation efficiency and specificity using a flow cytomer analysis. $10^{2}$ to $10^{5}$ labelled MCF7 cells in repeated assay were spiked in the prepared blood sample the results are shown below in figure. 7(A,B). We observed high separation efficiency in MCF7 cell separation from the sample. Around $83 \%$ overall separation efficiency was achieved.
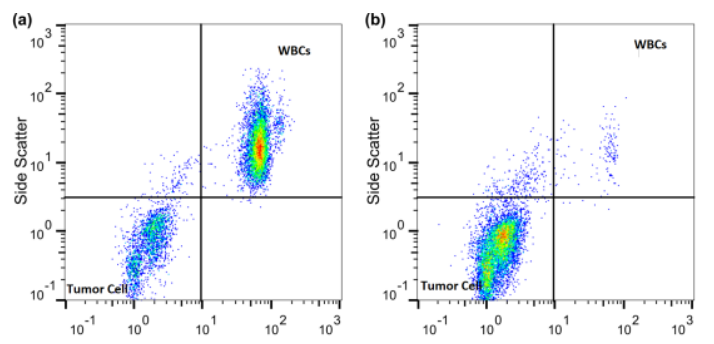

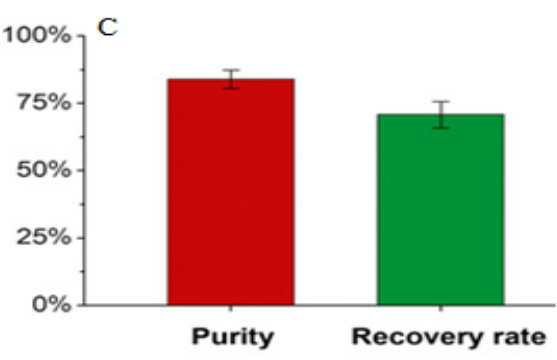

Figure 7. Flow cytometry results of (a) the mixture sample (WBCs mixed with tumor Cell), (b) separated tumor Cell sample collected through the lower outlet.

Additionally, the collected sample cells were stained for EpCAM, CD45, and DAPI are used to identify MCF7 from leukocytes and for separated cell purity determination. The purity was almost about $87 \%$ as in figure $.7(\mathrm{C})$.

\section{Conclusion}

Here we described the principles and experimental results of microfluidic technologies for cancer cell separation. The microfluidic devices are based on magnetic activated cell sorting. We fabricated and designed a simple lowcost chip for separation of CTCs from buffer/sample solution. We computationally simulated the external static magnetic intensity using modelling software COMSOL Multiphysics 4.3a (COMSOL, Inc.). The designed micro-device with permanent magnets array isolates the CTCs into the lateral -exit of the flow in the micro-channel. The micro- device was shown experimentally to separate around $87 \%$ of the spiked CTCs cancer cells (MCF-7). Microfluidic devices with an external permanent magnets array combined with a lab made fluidic control system are very useful for the precise and reliable microfluidic separation of CTCs as an integrated diagnostic instrument. Since the described designed micro-device protocols are quite simple and mass-producible, the microdevice can provide a useful and practical tool for allowing the micro-device to be costiveness producible as clinical tool for cancer diagnosis, prognosis, and personalized medicine.

\section{Acknowledgements}

This work in the school of life science, Beijing institute of technology and financially was was supported by the National Key Scientific Instrument and Equipment Development Project of China (No. 2012YQ040140).

\section{References}

1. W.-W. Sun, Z.-H. Xu, P. Lian, B.-L. Gao, and J.-A. $\mathrm{Hu}$, "Characteristics of circulating tumor cells in organ metastases, prognosis, and $\mathrm{T}$ lymphocyte mediated immune response," Onco. Targets. Ther., vol. Volume 10, pp. 2413-2424, May 2017. 
2. H. W. Hou, Q. S. Li, G. Y. H. Lee, A. P. Kumar, C. N. Ong, and C. T. Lim, "Deformability study of breast cancer cells using microfluidics," Biomed. Microdevices, vol. 11, no. 3, pp. 557-564, Jun. 2009.

3. M. Aghaamoo, Z. Zhang, X. Chen, and J. Xu, "Deformability-based circulating tumor cell separation with conical-shaped microfilters: concept, optimization and design criteria."

4. "Characteristics of circulating tumor cells (CTCs) in patients with lung cancer." [Online]. Available: https://www.mdlinx.com/oncology/conferenceabstract.cfm/57200/?nonus $=0 \&$ searchstring $=\&$ cover age_day $=0 \&$ page $=2$. [Accessed: 03-Oct-2017].

5. C. $\bar{W}$. Shields IV, J. L. Wang, K. A. Ohiri, E. D. Essoyan, B. B. Yellen, A. J. Armstrong, and G. P. López, "Magnetic separation of acoustically focused cancer cells from blood for magnetographic templating and analysis," Lab Chip, vol. 16, no. 19, pp. 3833-3844, Sep. 2016.

6. X. Mao, J. R. Waldeisen, and T. J. Huang, "Microfluidic drifting'-implementing three- dimensional hydrodynamic focusing with a singlelayer planar microfluidic device," Lab Chip, vol. 7, no. 10 , p. 1260 , Sep. 2007.

7. J. Darabi and C. Guo, "On-chip magnetophoretic isolation of $\mathrm{CD} 4+\mathrm{T}$ cells from blood.," Biomicrofluidics, vol. 7, no. 5, p. 54106, 2013.

8. J. Bartram and R. Ballance, "Water Quality Monitoring -A Practical Guide to the Design and Implementation of Freshwater Quality Studies and Monitoring Programmes Edited Chapter 11 BIOLOGICAL MONITORING,” pp. 0-419.

9. S. F. Ibrahim and G. van den Engh, "Flow Cytometry and Cell Sorting," in Advances in biochemical engineering/biotechnology, vol. 106, 2007, pp. 19-39.

10. C.-L. Chang, C. A. Savran, S. Jalal, and D. E. Matei, "Micro-aperture chip system for high-throughput immunomagnetic cell detection," in 2012 IEEE Sensors, 2012, pp. 1-4. 